\title{
Incorporación de la aplicación Kahoot! para la evaluación de las prácticas de la asignatura de "Ciencia de Materiales"
}

\section{Garcia-Garcia ${ }^{a}$, A. Carbonell-Verdu ${ }^{a}$, N. Montañes ${ }^{a}$, L. Quiles ${ }^{a}$ y V. Fombuena ${ }^{a}$}

${ }^{a}$ Universitat Politècnica de València. Departamento de Ingeniería Mecánica y de Materiales. Plaza Ferrándiz y Carbonell, 03801 Alcoy (Alicante) España. e-mail: dagarga4@epsa.upv.es

\begin{abstract}
During the accomplishment of the "Material science" laboratory practices in the Degree of Chemical Engineering, we have incorporated the use of the multi-platform application "Kahoot!" to evaluate the subject. The incorporation of this application improves the attention of the student during the development of the laboratory practices in an efficient and dynamic way. Furthermore, the application allows individual participation of students and debate between them in a dynamic and entertaining mode. The application improves student collaboration and attention in the development of the practices. The implementation of the multi-platform application "Kahoot!" encourages the attention and participation of the student in the laboratory practices in a very effective way.
\end{abstract}

Keywords: Gamification, methodology, applications, Kahoot!, game, competition, training, evaluation, mobile device.

\footnotetext{
Resumen

Durante la realización de las prácticas de laboratorio de la asignatura de "Ciencia de Materiales" en el Grado de Ingeniería Química se ha incorporado el uso de la aplicación multiplataforma Kahoot! para su evaluación. Con la incorporación de esta aplicación se consigue incrementar la atención del alumno de forma efectiva y dinámica durante el desarrollo de las mismas. Además la aplicación permite tanto la participación individual como el debate entre los propios alumnos de manera dinámica y entretenida, mejorando la colaboración y la atención del alumno en el transcurso de las prácticas. Con la implementación de la aplicación multiplataforma Kahoot! se fomenta la atención y participación del alumnado en las clases prácticas de una forma muy efectiva.
}

Palabras clave: gamificación, metodología, aplicaciones, Kahoot!, juego, competición, formación, evaluación, dispositivo móvil. 


\section{Introducción}

El gran avance tecnológico de los últimos años, así como el fácil acceso de los alumnos a dispositivos electrónicos ha dado lugar a nuevas formas de enseñanza en el aula [1]. Con el paso de los años, la aparición de nuevas técnicas ha facilitado a los docentes el gran reto de conseguir promover tanto la atención como la colaboración del alumno en clase. Incluso, permiten conocer rápidamente y de una manera mucho más efectiva, que métodos tradicionales como las preguntas de respuesta oral lanzadas a todo el grupo, si el alumnado está comprendiendo los conceptos y está realizando, por consiguiente, un correcto seguimiento de la asignatura [2].

Inicialmente, los sistemas de evaluación de respuesta personal (Clickers) obligaban a los centros a realizar inversiones en la adquisición de dispositivos de mano y equipos para que el alumno pudiera responder a las preguntas planteados por el profesor [3]. En la actualidad herramientas de trabajo como la aplicación multiplataforma Kahoot! están facilitando el aprendizaje y la evaluación en red con la utilización de los dispositivos electrónicos personales.

La aplicación Kahoot! es una herramienta evaluativa de carácter lúdico que permite realizar la misma función que los "Clickers" de una manera más sencilla [4]. Es un juego divertido y dinámico que consiste en proyectar una a una las preguntas sobre la pantalla del aula y el alumno con la utilización de un dispositivo electrónico, conectado previamente a internet, tratará de seleccionar la respuesta que crea correcta [5]. El alumno será puntuado distintamente dependiendo de la respuesta seleccionada y el tiempo empleado para ello. Al finalizar cada pregunta, el profesor aprovecha para generar un debate sobre la respuesta, involucrando al alumno en el proceso de aprendizaje [6]. Además, presenta ventajas como repasar los errores más comunes, conocer al alumnado con mayores dificultades y tener una visión general del alcance de conocimientos de una manera muy rápida y fácil.

Existen multitud de opciones para la evaluación mediante cuestionarios, un ejemplo es el caso de los exámenes tipo test disponibles en la plataforma "PoliformaT" de la UPV. Sin embargo, existen notables diferencias en cuanto a la dinámica de evaluación y la retroalimentación alumno-profesor de dichos cuestionarios con respecto a la aplicación Kahoot!. El principal inconveniente de dichos test es que están pensados para que el alumno conteste a todas las preguntas en un determinado tiempo, obteniendo la solución de todas ellas una vez finalizado el test, lo cual impide la posibilidad de retroalimentación con el profesor en cada pregunta, sin embargo, mediante Kahoot! el profesor puede interactuar con los alumnos tras cada cuestión realizada. Otra de las características que diferencian los cuestionarios habituales de la aplicación Kahoot! es la ausencia de competición entre los alumnos, omitiendo así la parte lúdica que sí se encuentra en Kahoot!.

En las encuestas y diferentes estudios consultados en bibliografía referentes a la utilización de los nuevos métodos interactivos, Kahoot! ha sido evaluado por parte del alumnado positivamente tanto en diversión de la actividad como utilidad [4].

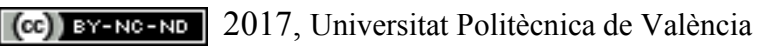




\section{Objetivos}

El objetivo principal del presente estudio es valorar la implementación de la aplicación multiplataforma Kahoot! en la evaluación de las prácticas de la asignatura de "Ciencia de Materiales" de segundo curso del Grado de Ingeniería Química. Con la incorporación de esta aplicación para la evaluación de la asignatura no se desea únicamente evaluar al alumno, sino que también se pretende mejorar la atención de los alumnos en clase, así como afianzar los conceptos importantes de la misma de una forma divertida y utilizando las nuevas tecnologías.

\section{Desarrollo}

Como se ha comentado, se ha introducido la aplicación Kahoot! como método de evaluación de las prácticas de la asignatura de "Ciencia de Materiales" de segundo curso del Grado de Ingeniería Química. En total se han realizado cinco pruebas con dicha aplicación (una por cada una de las sesiones de prácticas) a un total de 30 alumnos divididos en dos grupos. Para poder implementar dicha aplicación en las prácticas de la asignatura es necesario que cada alumno disponga de un dispositivo electrónico (móvil, tableta u ordenador) conectado a internet. También es necesario que en cada uno de los dispositivos esté instalada la aplicación Kahoot!, exceptuando en los ordenadores, ya que en estos se puede acceder a la aplicación directamente a través de la web.

\subsection{Preparación de los test de evaluación}

Para poder evaluar a los alumnos mediante la aplicación Kahoot!, en primer lugar el profesor creó cinco test (uno por cada sesión práctica de la asignatura) con 20 preguntas cada uno. Esto se realizó con antelación al inicio de las clases a través de la web de la aplicación (www.create.kahoot.it/). En dicha web el profesor puede crear una plantilla con las preguntas que desea realizar (Fig.1), además debe proponer diferentes soluciones a las preguntas planteadas, siendo al menos una de ellas la correcta, ya que la aplicación consiste en seleccionar la respuesta correcta dentro de las soluciones propuestas. Una vez preparados los test con las preguntas y las posibles soluciones ya se puede realizar la evaluación de cada una de las prácticas. Los test creados pueden ser guardados, lo cual permite su utilización para posteriores cursos. 


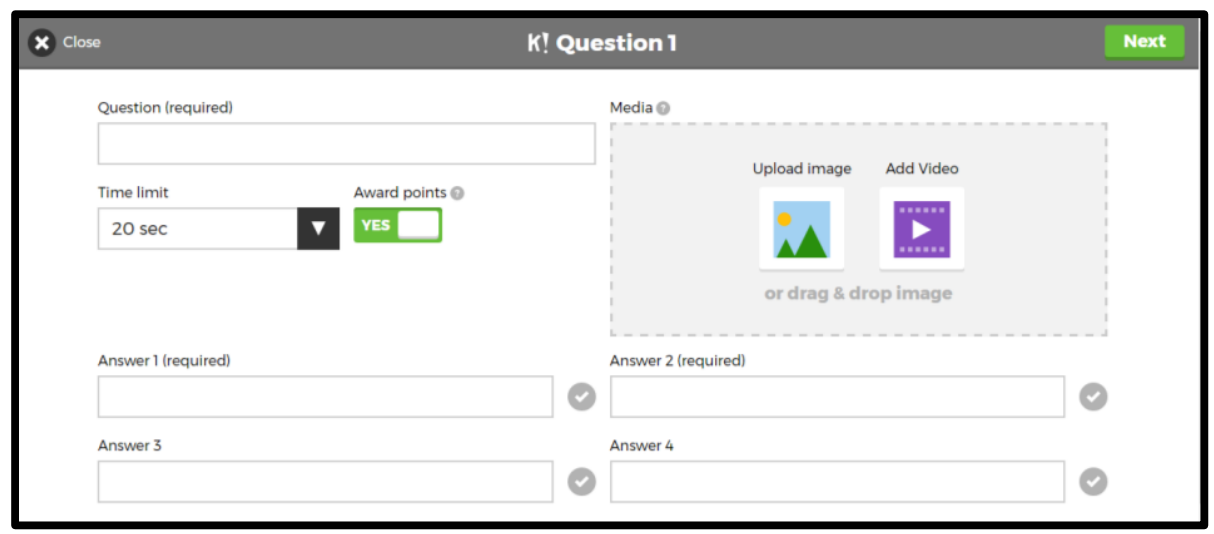

Fig. 1 Plantilla para la creación de preguntas en la aplicación Kahoot!

\subsection{Aplicación de los test de evaluación}

En este caso se consideró que el mejor momento para realizar la evaluación mediante Kahoot! era el inicio de cada una de las cinco sesiones prácticas, esto permite refrescar conceptos dados en clases anteriores y obliga al alumno a llevar un seguimiento constante de la asignatura. Para evitar que el inicio de la clase se alargase demasiado se dotó de un límite de tiempo para responder a cada una de las preguntas (20 segundos para cada pregunta). Para llevar a cabo la evaluación, en primer lugar el profesor debe de indicar a los alumnos el código del test, el cual proporciona la aplicación (Fig. 2). Una vez todos los alumnos han entrado en la aplicación deben identificarse mediante su nombre y apellidos, apareciendo todos los participantes en la pantalla del proyector.

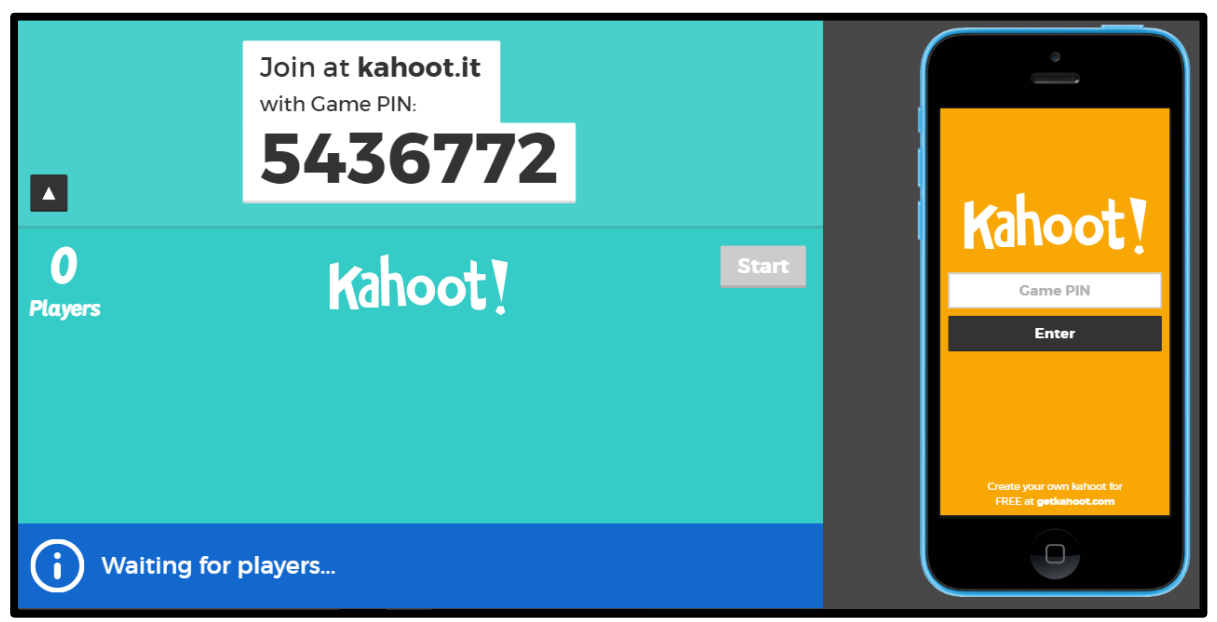

Fig. 2 Código del test proporcionado por la aplicación Kahoot! 
Cuando todos los alumnos están identificados el profesor inicia el test. Las preguntas con las posibles respuestas aparecen una tras otra en el proyector de clase y el alumno a través del dispositivo móvil dispone de un límite de tiempo para seleccionar la respuesta que consideren correcta (Fig. 3).

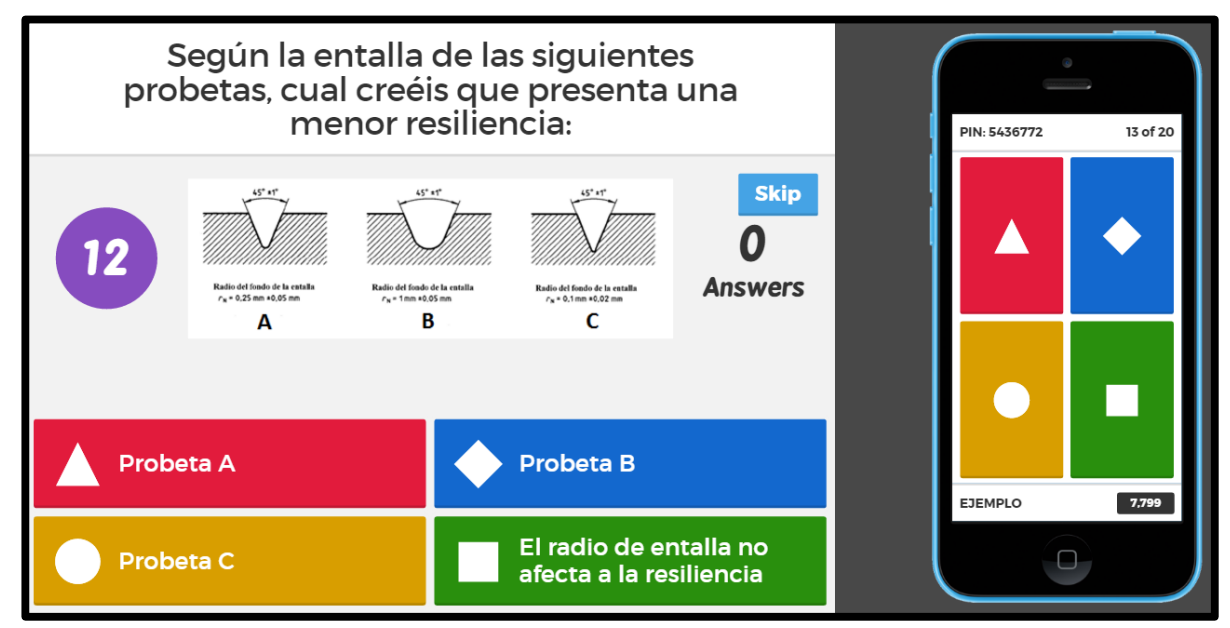

Fig. 3 Ejemplo de pregunta en la aplicación Kahoot!

Una vez finalizado el tiempo de la pregunta o cuando todos los alumnos hayan contestado a la misma, la aplicación dota a cada uno de ellos de una puntuación, la cual depende de si la respuesta seleccionada es la correcta y del tiempo que hayan empleado en seleccionarla. La aplicación también dota de puntos extra al alumno que haya acertado varias preguntas de forma consecutiva. Tras cada pregunta el nombre de los alumnos con las mayores puntuaciones aparece en la pantalla, motivando al alumno a superarse en preguntas posteriores (Fig. 4). Las preguntas planteadas en cada inicio de clase estarán relacionadas con las prácticas de laboratorio realizadas anteriormente, con el objetivo de refrescar conceptos ya impartidos y de evaluar lo aplicado en sesiones anteriores. 


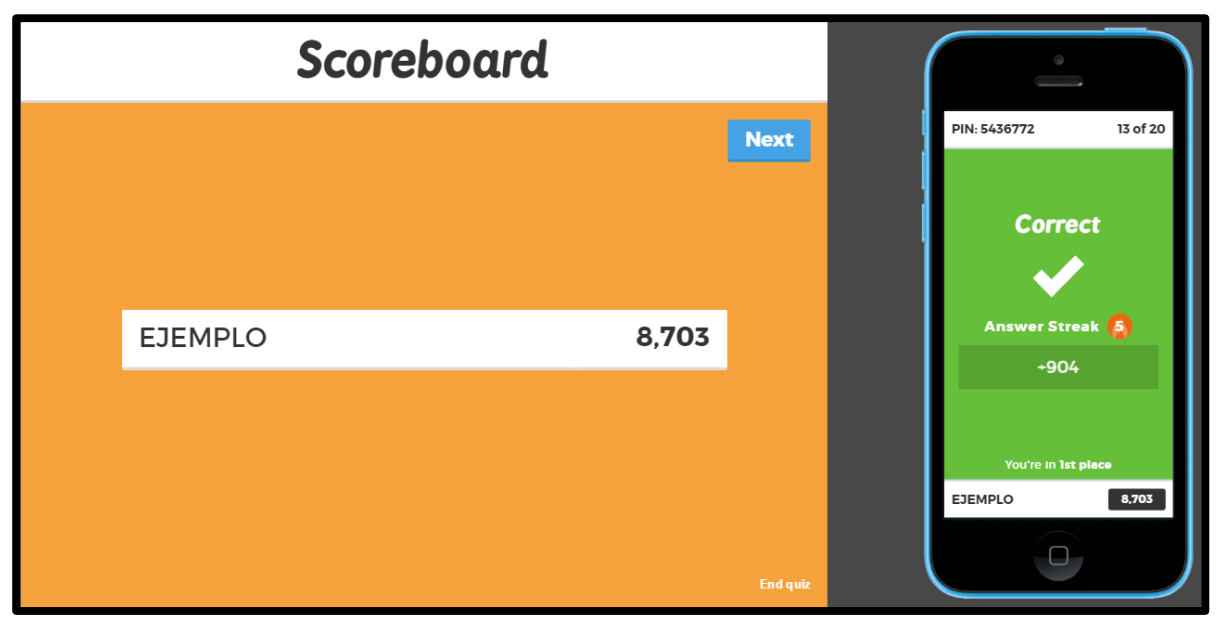

Fig. 4 Pantalla de resultados de la aplicación Kahoot!

\subsection{Evaluación de los test}

Una vez finalizado el test la aplicación permite descargar en el ordenador del profesor una hoja Excel con los resultados obtenidos por cada uno de los alumnos participantes, sabiendo las preguntas acertadas y falladas por cada uno de ellos (Fig. 5). Esto permite evaluar el test realizado de forma individual así como dotar de una puntuación numérica (del 0 al 10) a cada alumno de una forma objetiva. Tras finalizar los cinco cuestionarios el profesor dispuso de varias notas numéricas de cada uno de los alumnos permitiendo realizar una media para así obtener una nota final, la cual tuvo un peso específico del $20 \%$ en la nota final de la asignatura. Para motivar al alumno, se otorgó la máxima puntuación de cada test (un 10) a los cinco alumnos con la mayor puntuación obtenida en cada test, independientemente del número de fallos que cometieron, el resto de alumnos fue valorado teniendo en cuenta el número de aciertos y de fallos de cada test. Esta aplicación no sólo permite evaluar al alumno de forma numérica sino que también proporciona al profesor un conocimiento sobre el seguimiento de la asignatura por parte de cada alumno. 


\begin{tabular}{|c|c|c|c|c|c|c|c|}
\hline 4 & A & & $B$ & & C & $\mathrm{D}$ & $\mathrm{E}$ \\
\hline 1 & Prá & cas Ciencia de & Aateriales & & & & \\
\hline 2 & Final & ores & & & & & \\
\hline 3 & Rank & Players & & Total Sco & re (points) & Correct Answers & Incorrect Answers \\
\hline 4 & & 1 Manu Cartagena & & & 13106 & 14 & 6 \\
\hline 5 & & 2 raquel & & & 12313 & 13 & 7 \\
\hline 6 & & 3 Cami & & & 11837 & 12 & 8 \\
\hline 7 & & 4 Silvia & & & 11119 & 12 & 8 \\
\hline 8 & & 5 Andrés & & & 10076 & 11 & 9 \\
\hline 9 & & 6 Maria & & & 9939 & 11 & 8 \\
\hline 10 & & 7 Jordina & & & 9599 & 11 & 8 \\
\hline 11 & & 8 Mar lópez & & & 8195 & 10 & 9 \\
\hline 12 & & 9 irene & & & 7918 & 9 & 11 \\
\hline 13 & & 10 Luis & & & 6321 & 7 & 13 \\
\hline 4 & ' & Final Scores & Question Summary & Question 1 & Question 2 & .. $\oplus: 4$ & \\
\hline
\end{tabular}

Fig. 5 Hoja Excel con los resultados de una de las pruebas

\section{Resultados}

Tras finalizar las cinco sesiones prácticas de la asignatura de "Ciencia de Materiales" se realizó una encuesta anónima a los 30 alumnos que cursaron la asignatura con el fin de que evaluaran el método de evaluación empleado en las prácticas, obteniéndose los resultados que se muestran en la Fig. 6.

Tabla 1. Resultados de la encuesta sobre la metodología de evaluación

\begin{tabular}{ccccc}
\hline & \multicolumn{4}{c}{ VALORACIÓN } \\
\cline { 2 - 4 } Cuestión & $\begin{array}{c}\text { Nada } \\
\text { Favorable }\end{array}$ & $\begin{array}{c}\text { Poco } \\
\text { Favorable }\end{array}$ & Favorable & $\begin{array}{c}\text { Muy } \\
\text { Favorable }\end{array}$ \\
\hline $\begin{array}{c}\text { ¿Qué te ha parecido el sistema } \\
\text { de evaluación de las prácticas } \\
\text { de "Ciencia de Materiales" } \\
\text { mediante la aplicación } \\
\text { Kahoot!? }\end{array}$ & 0 & 0 & 7 & 23 \\
\hline
\end{tabular}

Como se puede observar a más de un $76 \%$ le pareció muy favorable el método de evaluación empleado, mientras que al $24 \%$ restante le pareció favorable. Por tanto, se deduce que el sistema de evaluación de las prácticas mediante la aplicación Kahoot! resulta totalmente satisfactorio para el alumnado.

Además, también se observó un incremento de la nota media de las prácticas con respecto a los dos últimos cursos (2014/2015 y 2015/2016) donde la evaluación se realizó mediante informes individuales de cada una de las cinco sesiones de prácticas. Como se puede ver en 
la Fig. 5, tras la introducción de la aplicación multiplataforma Kahoot! como sistema de evaluación se obtuvo un incremento de la nota media superior al $12 \%$ con respecto al curso académico anterior (2015/2016). Dicho incremento de la nota media puede ser debido a un aumento de la atención en clase por parte del alumno debido al sistema de evaluación, así como a la mayor objetividad en la evaluación de las prácticas que permite la aplicación Kahoot! con respecto a los informes tradicionales. Además, como se muestra en la Tabla 1, la satisfacción del sistema de evaluación por parte de los alumnos, principalmente debido a su carácter lúdico y competitivo puede influir de forma positiva en el aumento de la nota media.

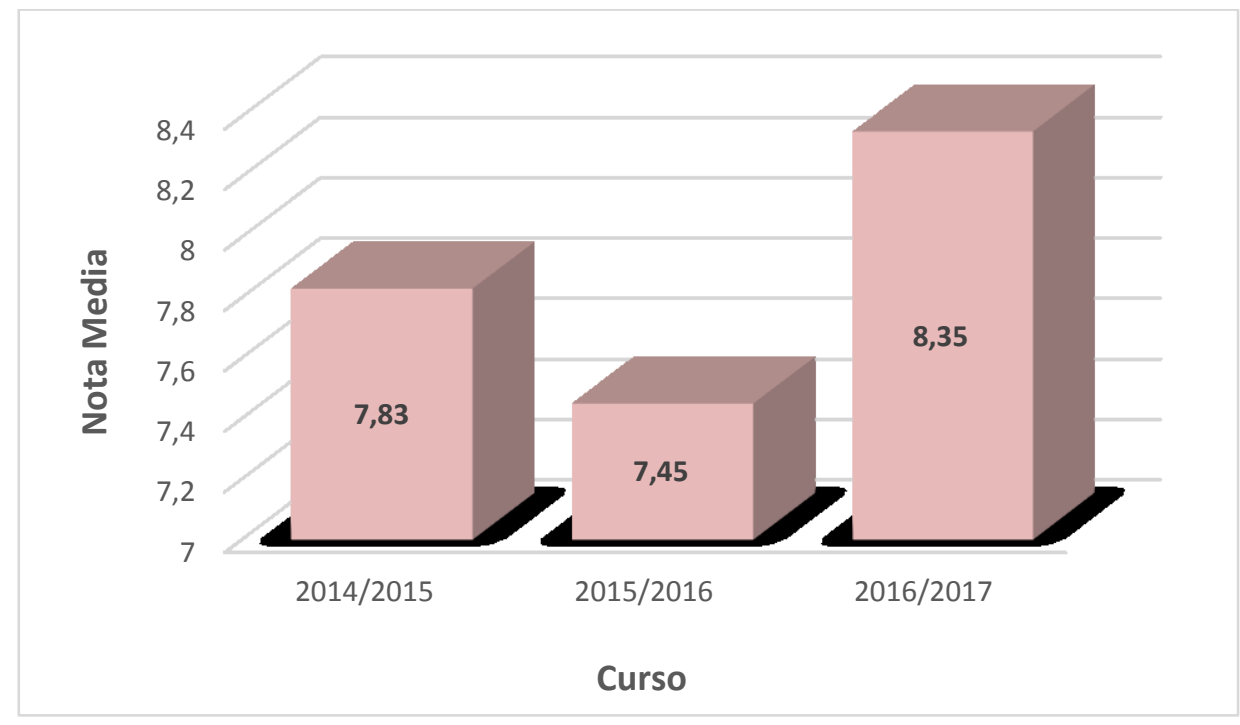

Fig. 5 Nota media de las prácticas de la asignatura de "Ciencia de Materiales"

\section{Conclusiones}

El empleo de la aplicación Kahoot! para la evaluación de las prácticas de laboratorio de las asignaturas resulta de gran interés ya que mediante este sistema de evaluación se consigue calificar al alumno de forma individual y se evita de esta manera el copiado de los informes de prácticas tan habitual. Además mediante este sistema el profesor puede tener un mayor seguimiento de cada uno de los alumnos. Con la implementación de este sistema de evaluación también se ha observado un aumento de la atención del alumno en clase así como una mayor participación de los mismos en las prácticas. Este tipo de aplicación también favorece la asimilación de conceptos fundamentales por parte del alumno y todo esto se consigue de una forma amena y divertida con el empleo de las nuevas tecnologías. Uno de los principales inconvenientes de la aplicación es la posible salida de algún alumno del test en curso debido a problemas de conexión a la red, lo cual le excluye de la evaluación final mediante la aplicación, este tipo de inconvenientes debería ser uno de los puntos débiles a mejorar por la aplicación.

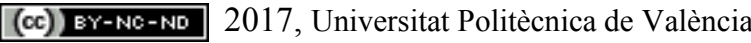




\section{Referencias}

[1] WANG, AI (2015). "The wear out effect of a game-based student response system" en Computers \& Education, vol. 82, p. 217-227.

[2] CEMBREROS, Diana (2016). “Prevención de errores frecuentes en Inglés mediante sistemas electrónicos de respuesta personal” en Revista de Ciencias Humanas y SocialesEspecial, vol. 11, p. 282-295.

[3] ROIG-VILA, R. et al (2016). Investigación e Innovación Educativa en Docencia Universitaria. Retos, Propuestas y Acciones. Alicante: Universidad de Alicante. Instituto de Ciencias de la Educación.

[4] HOlguíN, E., GARGANTILla MADERA, P., HERREROS RUIZ-VALDEPEÑAS., B. y LÓPEZ DEL HIERRO, M. (2015). "Kahoot en docencia: una alternativa práctica a los clickers" en XI Jornadas Internacionales de Innovación Universitaria Educar para transformar. Universidad Europea de Madrid. Disponible en: <http:/hdl.handle.net/11268/3603> [Consulta: 30 de marzo de 2017].

[5] BICEN, H y KOCAKOYUN, S. (2017). "Determination of university students' most preferred mobile application for gamification" en World Journal on Educational Technology: Current Issues, vol. 9, issue 1, p. 18-23.

[6] ALBA FERRE, E., MORENO BLESA, L. y RUIZ GONZALEZ, M. "The Star System apps to bridge educational gaps: Kahoot, Screencast y tableta gráfica” en XII Jornadas Internacionales de Innovación Universitaria Educar para transformer:aprendizaje experiencial. Universidad Europea de Madrid. Disponible en: < http://hdl.handle.net/11268/4493> [Consulta: 30 de marzo de 2017]. 